\title{
Analysis of two-dimensional combustion waves arising in the presence of a competitive endothermic reaction
}

\author{
$\begin{array}{lll}\text { S. D. Watt } & \text { Z. Huang } & \text { H. S. Sidhu } \\ & \end{array}$
}

(Received 27 February 2019; revised 21 May 2019)

\begin{abstract}
We consider a system of reaction-diffusion equations describing combustion dynamics. The reaction is assumed to undergo two competitive reactions, one which is exothermic and one which is endothermic. The one-dimensional model has been shown to exhibit complex behaviour, from propagating combustion waves with a constant speed to period doubling cascades and the possibility of chaotic wave speeds. In this study, we extend the combustion model from one to two dimensions by exploring a model of an insulated strip with no heat loss and axially symmetric spread. In particular, we compare and contrast the behaviour of the systems in one and two dimensions.
\end{abstract}

DOI:10.21914/anziamj.v60i0.14065 gives this article, (c) Austral. Mathematical Soc. 2019. Published July 15, 2019, as part of the Proceedings of the 18th Biennial Computational Techniques and Applications Conference. ISSN 1445-8810. (Print two pages per sheet of paper.) Copies of this article must not be made otherwise available on the internet; instead link directly to the DOI for this article. 


\section{Contents}

1 Introduction

C96

2 Mathematical model

2.1 Competitive reactions in a strip . . . . . . . . . . C98

2.2 Competitive reactions with axial symmetry . . . . . . C99

2.3 Numerical solution of problem in 2D strip . . . . . . . C100

3 Travelling wave solutions

4 Comparison between combustion in a 2D strip and in other configurations

C101

5 Critical conditions on combustion in a 2D strip

C102

6 Conclusion

C105

\section{Introduction}

The modelling of the ignition process of a combustible fuel can involve complex mechanisms and a large number of reactions. One of the simplifying assumptions is to group all of the reactions into a small number of reactions. In this work, we consider systems where there are two reactions competing for the consumption of the fuel source, one exothermic and one endothermic.

When modelling combustion processes, the success or failure depends on many factors. In this study, we model the combustion process in a two-dimensional strip with insulated boundaries. If the strip is too narrow, then the combustion process will not properly develop and the temperature will diffuse away [1]. Similarly, if the initial temperature profile does not provide enough energy, then the combustion process will also fail [6]. We explore the effect the Lewis 
number, a ratio of the thermal and mass diffusivities, has on the combustion process.

\section{Mathematical model}

We consider a system of reaction-diffusion equations describing combustion dynamics similar to the model proposed by Hmaidi, McIntosh, and Brindley [3] and analysed by Sharples et al. [5]. We assume that the reactant undergoes two competitive reactions, one exothermic and one endothermic. Arhhenius kinetics are assumed for both reactions, with the endothermic reaction characterised by the activation energy $E_{1}$, the pre-exponential factor $A_{1}$ and heat release $-Q_{1}<0$. The exothermic reaction drives the combustion and is characterised by the activation energy $E_{2}$, the pre-exponential factor $A_{2}$ and heat release $\mathrm{Q}_{2}>0$.

The governing equations for the two-dimensional system described above are the heat and mass balance equations accounting for reaction and diffusion of heat and reactant:

$$
\begin{aligned}
\rho c_{p} \frac{\partial T}{\partial t} & =k \nabla^{2} T+\rho\left(-Q_{1} A_{1} e^{-E_{1} / R_{0} T}+Q_{2} A_{2} e^{-E_{2} / R_{0} T}\right) C, \\
\rho \frac{\partial C}{\partial t} & =\rho D \nabla^{2} C-\rho\left(A_{1} e^{-E_{1} / R_{0} T}+A_{2} e^{-E_{2} / R_{0} T}\right) C,
\end{aligned}
$$

where $T$ and $C$ are the temperature and reactant mass fraction, respectively, $t$ is the time coordinate, $\rho$ is the density, $k$ is the thermal conductivity, $c_{p}$ is the heat capacity at constant pressure of the reactant, $\mathrm{D}$ is the coefficient of mass diffusion, $R_{0}$ is the universal gas constant, and $\nabla^{2}$ is the Laplacian. Mercer and Weber [4] used similar equations. We consider a non-dimensional version of the system and introduce the dimensionless temperature, space and time coordinates

$$
u=\frac{R_{0} T}{E_{2}}, \quad x^{\prime}=\sqrt{\frac{\rho Q_{2} A_{2} R_{0}}{k E_{2}}} x, \quad y^{\prime}=\sqrt{\frac{\rho Q_{2} A_{2} R_{0}}{k E_{2}}} y, \quad t^{\prime}=\frac{Q_{2} A_{2} R_{0}}{c_{p} E_{2}} t .
$$


Writing the system in terms of these dimensionless quantities and omitting the primes, the dimensionless system of equations is

$$
\begin{aligned}
& \frac{\partial u}{\partial t}=\frac{\partial^{2} u}{\partial x^{2}}+\frac{\partial^{2} u}{\partial y^{2}}+C e^{-1 / u}-q r C e^{-f / u} \\
& \frac{\partial C}{\partial t}=\frac{1}{L e}\left(\frac{\partial^{2} C}{\partial x^{2}}+\frac{\partial^{2} C}{\partial y^{2}}\right)-\Theta C e^{-1 / u}-\Theta C r e^{-f / u}
\end{aligned}
$$

with new parameters

$$
\Theta=\frac{c_{p} E_{2}}{R_{0} Q_{2}}, \quad q=\frac{Q_{1}}{Q_{2}}, \quad f=\frac{E_{1}}{E_{2}}, \quad r=\frac{A_{1}}{A_{2}}, \quad L e=\frac{k}{\rho c_{p} D} .
$$

We refer to $\Theta$ as the exothermicity parameter, $q$ as the ratio of heats, $f$ as the ratio of activation energies, $r$ as the ratio of rate constants, and Le as the Lewis number.

In the following section we explore two different domain configurations and compare the behaviour of each configuration with the equivalent system in one dimension.

\subsection{Competitive reactions in a strip}

We first explore a long strip domain. Mercer and Weber [4] explored a similar problem with a one-step model. In addition to the system (3)-(4), we impose insulating boundary conditions on the left (which is assumed to be at $x=0$ ) and right boundaries

$$
\frac{\partial u}{\partial x}=0 \text { and } \quad \frac{\partial C}{\partial x}=0 \quad \text { at } x=0 \text { and } x \rightarrow \infty .
$$

We assume insulating boundaries at the top and bottom of the strip $(y=0$ and $y=H$ )

$$
\frac{\partial u}{\partial y}=0 \quad \text { and } \quad \frac{\partial C}{\partial y}=0
$$




\subsection{Competitive reactions with axial symmetry}

The second domain configuration is the infinite two-dimensional plane. However, we assume that the initial temperature profile is axially symmetric about a point (which is assumed to be at the origin). As the system parameters do not depend on the spatial coordinates, the solution retains the axial symmetry as time progresses. This allows the problem to be simplified by changing to a polar coordinate system

$$
x=R \cos \theta, \quad y=R \sin \theta,
$$

where $R$ is the radius from the origin and $\theta$ is the angle from the $x$-axis in an anticlockwise direction. The system then becomes

$$
\begin{aligned}
& \frac{\partial u}{\partial t}=\frac{\partial^{2} u}{\partial R^{2}}+\frac{1}{R} \frac{\partial u}{\partial R}+\frac{1}{R^{2}} \frac{\partial^{2} u}{\partial \theta^{2}}+C e^{-1 / u}-C_{\text {qre }}-f / u, \\
& \frac{\partial C}{\partial t}=\frac{1}{L e}\left(\frac{\partial^{2} C}{\partial R^{2}}+\frac{1}{R} \frac{\partial C}{\partial R}+\frac{1}{R^{2}} \frac{\partial^{2} C}{\partial \theta^{2}}\right)-\Theta C e^{-1 / u}-\Theta C r e^{-f / u} .
\end{aligned}
$$

Assuming axial symmetry, or no $\theta$ dependence, the system reduces to

$$
\begin{aligned}
& \frac{\partial u}{\partial t}=\frac{\partial^{2} u}{\partial R^{2}}+\frac{1}{R} \frac{\partial u}{\partial R}+C e^{-1 / u}-C_{q r e}-f / u \\
& \frac{\partial C}{\partial t}=\frac{1}{L e}\left(\frac{\partial^{2} C}{\partial R^{2}}+\frac{1}{R} \frac{\partial C}{\partial R}\right)-\Theta C e^{-1 / u}-\Theta C r e^{-f / u} .
\end{aligned}
$$

This system is valid for $R>0$. However, at $R=0$ we also need to impose the usual boundary condition that

$$
\frac{\partial u}{\partial R}=\frac{\partial C}{\partial R}=0 .
$$

The system with axial symmetry is numerically solved using the Method of Lines, where the spatial derivatives are approximated by a finite difference method and the resulting ODEs at each grid point are integrated using a Runge-Kutta solver. 


\subsection{Numerical solution of problem in 2D strip}

Figures 1, 2 and 3 show the time evolution of a Gaussian initial temperature in the two-dimensional strip, the axial symmetric two-dimensional strip, and the one-dimensional domain. In these figures, the time is slightly different for the one-dimensional plots to better align with the two-dimensional strip at $t=1900$. There are three stages of the evolution:

1. Initially, the temperature profile is axially symmetric and remains symmetric (see Figure 1).

2. In the medium term, the temperature profile begins interacting with the top boundary (see Figure 2).

3. In the long term, the temperature profile loses vertical spatial dependence and the behaviour is similar to the one-dimensional problem (see Figure 3).

\section{Travelling wave solutions}

One of the areas of interest in many combustion problems is the behaviour of propagating combustion waves. For one-dimensional problems, depending on the reaction processes, there is a rich variety of travelling wave behaviour. For different system parameters, there can be a transition of behavior. For example, Sharples et al. [5] showed that for the one-dimensional exothermicendothermic reaction there is a route from travelling waves with a single speed, to wave with an oscillatory speeds to a period doubling regime, to extinction. There are also studies of other reaction systems which observed a period doubling route to chaotic behaviour [2]. 
(a)
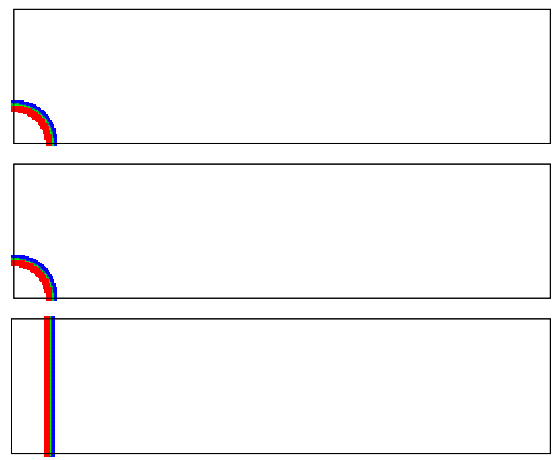

(b)
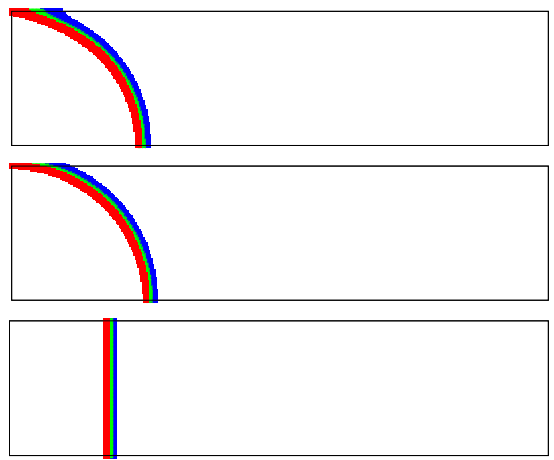

Figure 1: Evolution of Gaussian initial temperature profile in the three domain configurations for $\mathrm{Le}=5, \Theta=3, \mathrm{q}=1, \mathrm{r}=1$ and $\mathrm{f}=2$ at times (a) $t=0$ and (b) $t=400$ for the two-dimensional insulated strip of height $\mathrm{H}=100$ (upper frames) and axial symmetric domain (middle frames); and (a) $t=0$ and (b) $t=200$ for the one-dimensional domain (lower frames). Each domain is of length $L=400$ and height $H=100$. The blue, green and red colours represent the beginning, middle and end of the reaction zone.

\section{Comparison between combustion in a 2D strip and in other configurations}

Figure 4 compares the locations of the Hopf and extinction curves of the onedimensional model [5], the two-dimensional model and the axially symmetric model. The Hopf curve for the one-dimensional model is obtained by solving the associated ordinary difference equations using the Evans function method, while the extinction curve is obtained by directly solving the partial differential equations (PDE) using FlexPDE ${ }^{\mathrm{TM}}$ and finding the exothermicity value where no non-trivial solutions exist. There is good agreement between the Hopf curves of the two-dimensional and one-dimensional problems. For a long enough strip, the two-dimensional profile will evolve to a one-dimensional profile in the long term. There is fair agreement with the axially symmetric problem. There are slight differences in the locations of the extinction points 


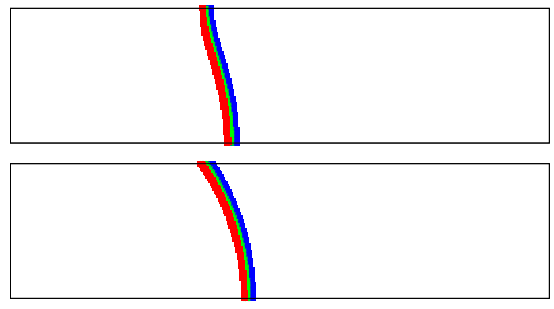

(a)

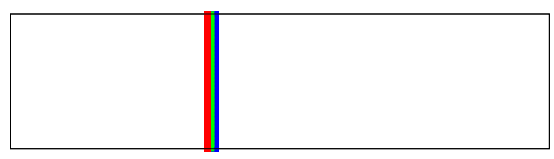

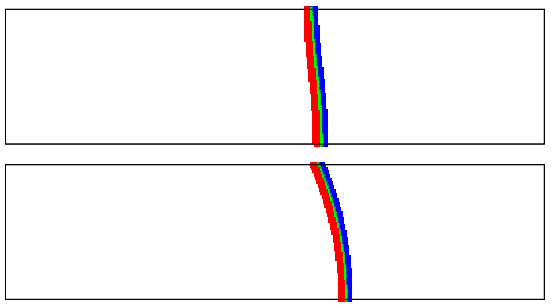

(b)

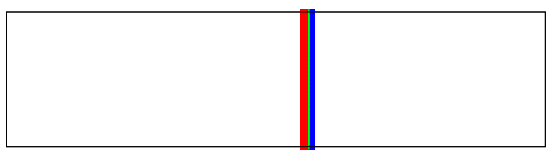

Figure 2: Evolution of Gaussian initial temperature profile in the three domain configurations for $\mathrm{Le}=5, \Theta=3, \mathrm{q}=1, \mathrm{r}=1$ and $\mathrm{f}=2$ at times (a) $t=800$ and (b) $t=1200$ for the two-dimensional insulated strip of height $\mathrm{H}=100$ (upper frames) and axial symmetric domain (middle frames); and (a) $t=600$ and (b) $t=1000$ for the one-dimensional domain (lower frames). Each domain is of length $L=400$ and height $H=100$. The blue, green and red colours represent the beginning, middle and end of the reaction zone.

in all three configurations. As mentioned by Watt et al. [6], the initial temperature profile is important in determining whether extinction will occur. The axially symmetric configuration requires a 'hotter' initial temperature profile than the other two configurations for combustion to be sustainable.

\section{Critical conditions on combustion in a $2 \mathrm{D}$ strip}

Section 4 shows that the behaviour of combustion in a strip is qualitatively similar to the problem in one dimension. Certainly, in the adiabatic problem considered here, a two-dimensional initial temperature profile quickly loses spatial variation with respect to height and then behaves according to the one-dimensional problem. 

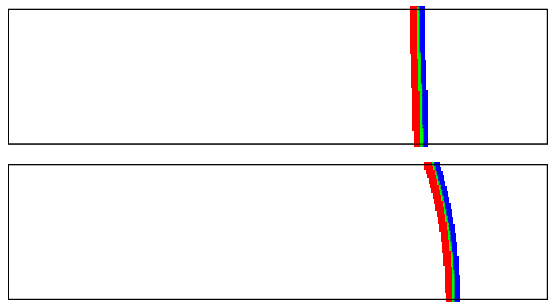

(a)

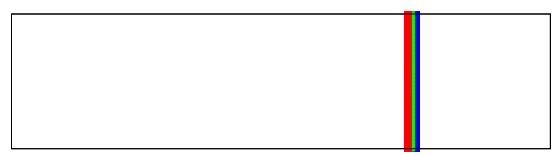

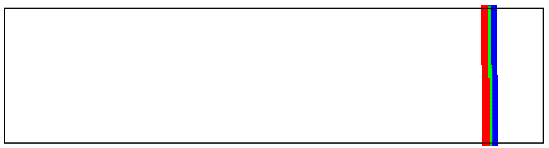

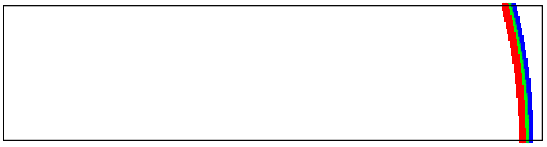

(b)

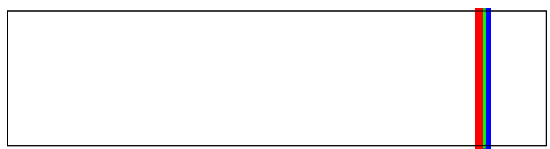

Figure 3: Evolution of Gaussian initial temperature profile in the three domain configurations for $\mathrm{Le}=5, \Theta=3, \mathrm{q}=1, \mathrm{r}=1$ and $\mathrm{f}=2$ at times (a) $t=1600$ and (b) $t=1900$ for the the two-dimensional insulated strip of height $\mathrm{H}=100$ (upper frames) and axial symmetric domain (middle frames); and (a) $t=1400$ and (b) $t=1700$ one-dimensional domain (lower frames). Each domain is of length $L=400$ and height $H=100$. The blue, green and red colours represent the beginning, middle and end of the reaction zone.

We explore the effect of the height of the strip $\mathrm{H}$ has on the behaviour of the full two-dimensional system (3)-(4). We choose parameters Le $=5, \Theta=5$, $\mathrm{L}=1000, \mathrm{H}=250, \mathrm{~A}=1.5$ and $\mathrm{b}=0.005$, with the initial temperature profile

$$
u(x, y)=A e^{-b\left(x^{2}+y^{2}\right)} .
$$

For these parameters, the one-dimensional system develops into a single speed propagating wave. The two-dimensional problem was solved numerically using FlexPDE ${ }^{\mathrm{TM}}$. In Figure 5 we see that there is good agreement between the extinction curve of the two-dimensional and axially symmetric problems.

We now vary the strip height $\mathrm{H}$ and we see in Figure 6 that the critical exothermicity quickly decays to an equilibrium value of $\Theta=4.48$, which is very close to the corresponding value of $\Theta=4.50$ for the axially symmetric problem. This suggests that for a sufficiently tall strip, the combustion wave develops as an axially symmetric wave before reaching the top of the strip. 

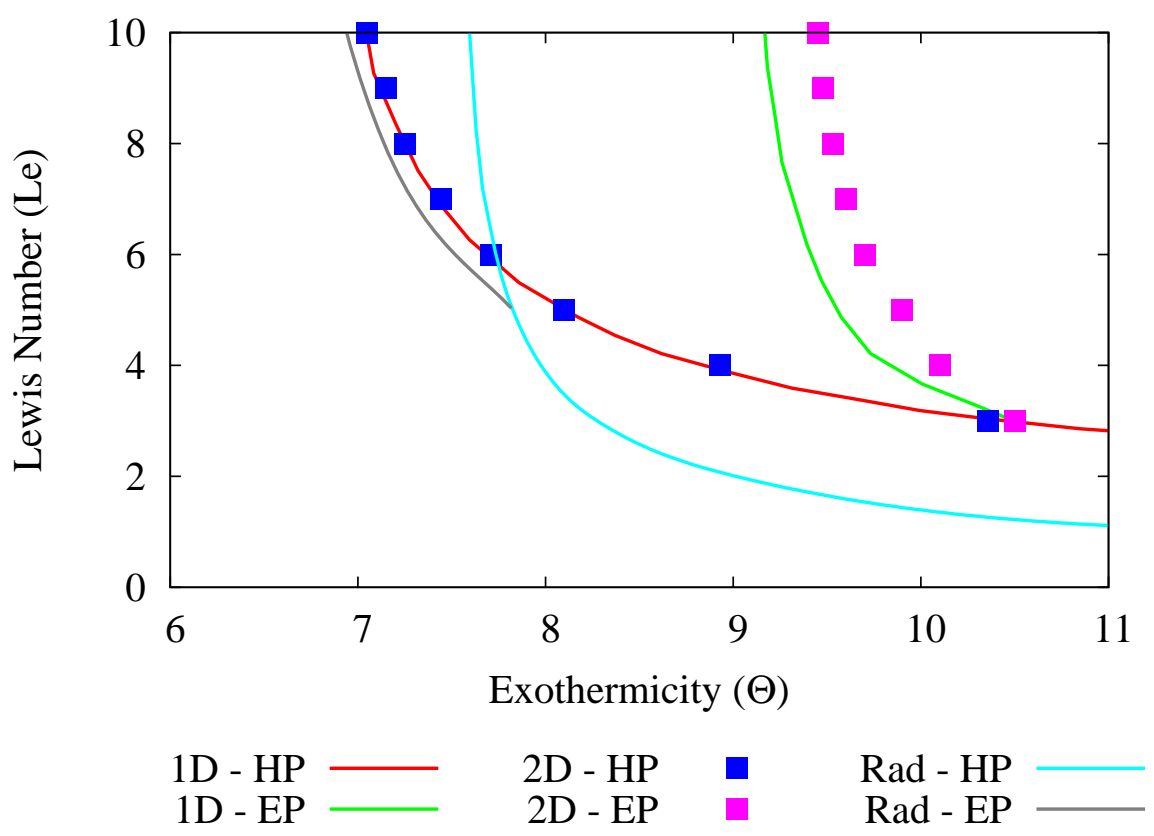

Figure 4: A comparison of the location of the Hopf point (HP) and the extinction point (EP) in one-dimensional (1D), two-dimensional strip (2D) and axially symmetric (Rad) configurations. The parameters are $\mathrm{H}=100$, $f=2, q=1$ and $r=1$.

Finally, we explore the critical initial temperature peak height $A$ for a combustion wave to propagate, as shown in Figure 7. Like Figure 6, the twodimensional and axially symmetric problems have similar behaviour. In Figure 7 there is a critical initial temperature peak height that is approached for large strip heights. For the two-dimensional problem, this critical height is at $A=2.72$, whereas for the axially symmetric problem, $A=2.69$. Note that the critical strip height is around $\mathrm{H}=400$ for the critical initial temperature peak height, whereas it was around $\mathrm{H}=200$ for the critical extinction exothermicity.

We do not explore the effect of parameters $f, q$ or $r$ on the long term behaviour 


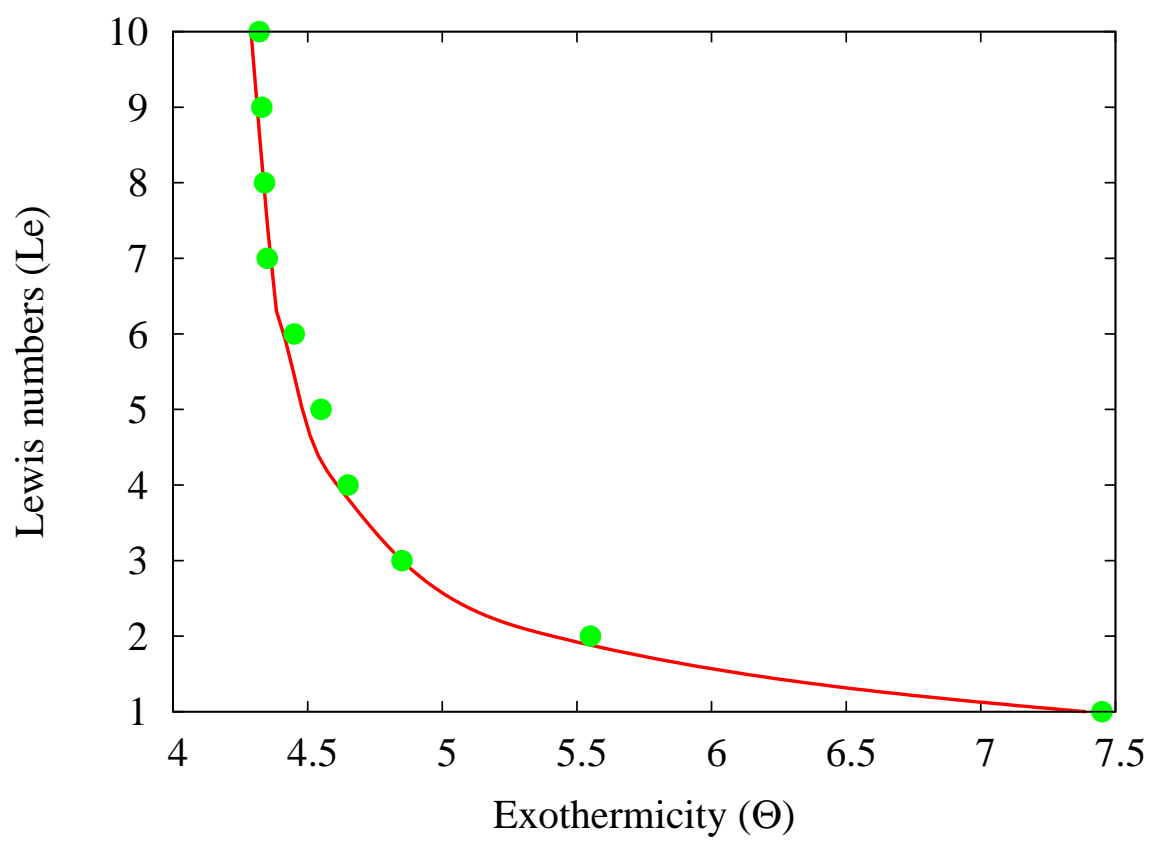

Figure 5: The extinction curve for the two-dimensional problem as a function of the Lewis number Le and exothermicity $\Theta$ with length $L=1000$, height $H=250$ and initial temperature peak height $A=1.5$. The green points represent the extinction curve for the axially symmetric problem.

of a two-dimensional strip with varying height $\mathrm{H}$. For the one-dimensional problem, the effect of these parameters on the persistence of a combustion wave solution was explored by others, such as Hmaidi, McIntosh, and Brindley [3] and Sharples et al. [5].

\section{Conclusion}

We considered the behaviour of combustion process of an exothermic-endothermic competitive reaction in a combustible material in a two-dimensional strip. 


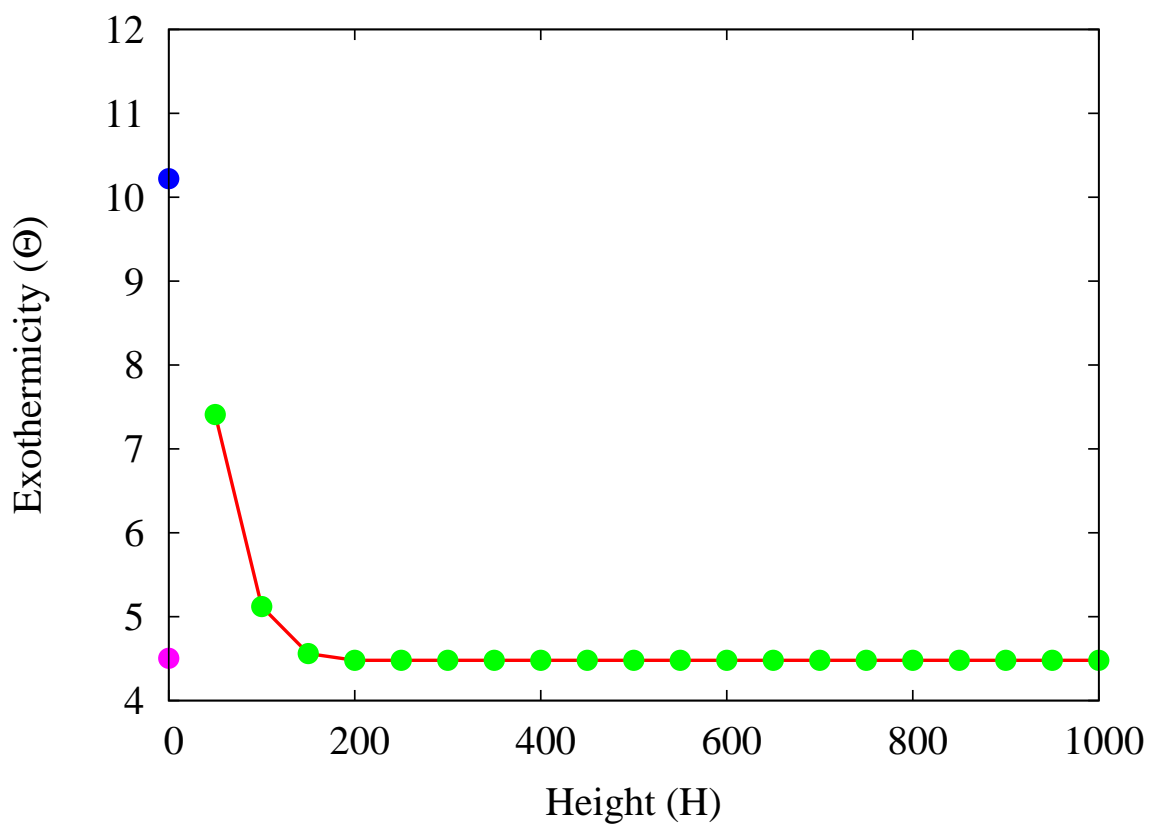

Figure 6: The extinction curve for the two-dimensional problem as a function of the strip height $\mathrm{H}$ with $\mathrm{Le}=5, \mathrm{~L}=1000$ and $A=1.5$. The blue and purple points are the corresponding extinction points for the one-dimensional and axially symmetric problems, respectively.

It was shown that for a combustion wave to persist, three of the important parameters are the height of the strip, the magnitude of the initial temperate profile and the ratio of the thermal and mass diffusivities defined by the Lewis number. The success or failure of the combustion wave to propagate is dependent on the state of the system once the combustion wave interacts with the top boundary. If there is sufficient energy in the combustion wave, then behaviour will follow the behaviour of an equivalent one-dimensional problem. 


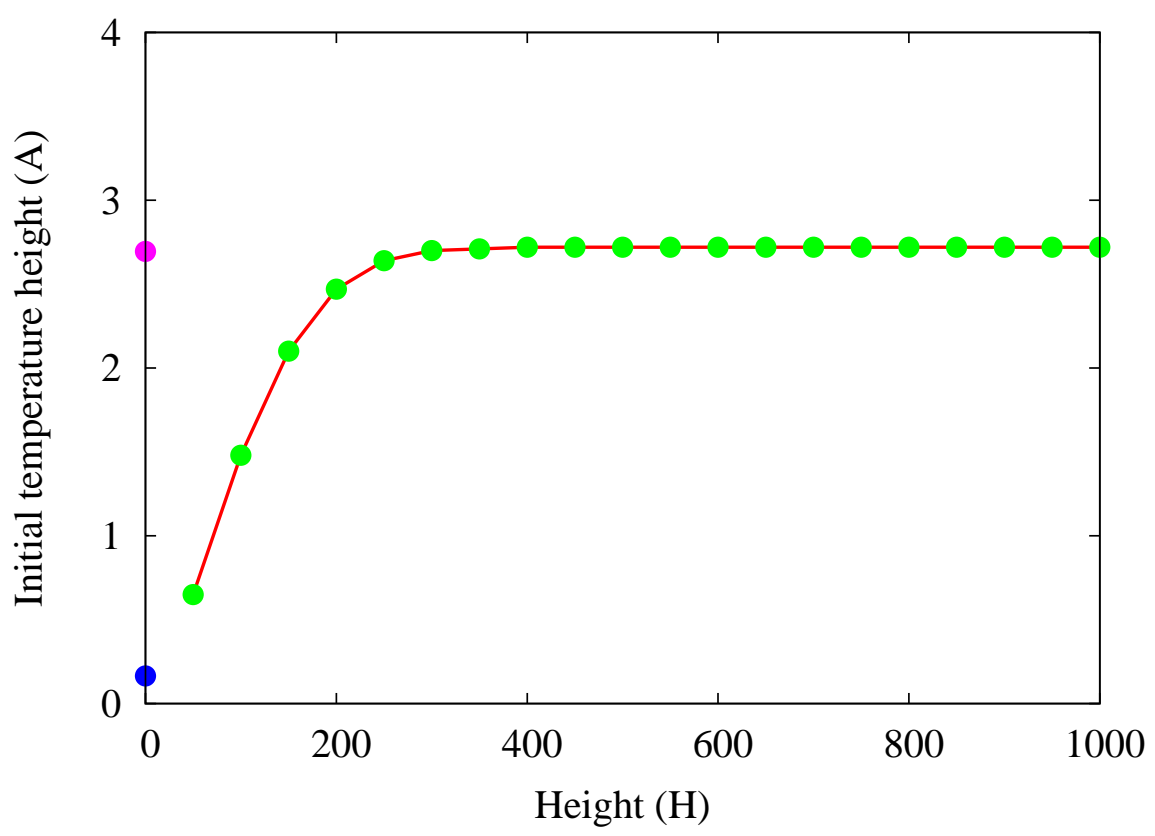

Figure 7: The critical initial temperature peak height $A$ for the twodimensional problem as a function of the strip height $\mathrm{H}$ with $\mathrm{Le}=5, \Theta=5$, $\mathrm{L}=1000$ and $A=1.5$. The blue and purple points are the corresponding critical initial condition heights for the one-dimensional and axially symmetric problems, respectively.

\section{References}

[1] J. D. Buckmaster and G. S. S. Ludford. Theory of Laminar Flames. Cambridge Monographs on Mechanics and Applied Mathematics. Cambridge University Press, 1982. DOI: 10.1017/CB09780511569531 (cit. on p. C96).

[2] V. Gubernov, A. Kolobov, A. Polezhaev, H. Sidhu, and G. Mercer. "Period doubling and chaotic transient in a model of chain-branching 
combustion wave propagation." In: P. Roy. Soc. A Math. Phy. 466 (2010), pp. 2747-2769. DOI: 10.1098/rspa. 2009.0668 (cit. on p. C100).

[3] A. Hmaidi, A. C. McIntosh, and J. Brindley. "A mathematical model of hotspot condensed phase ignition in the presence of a competitive endothermic reaction". In: Combust. Theor. Model. 14 (2010), pp. 893-920. DOI: 10.1080/13647830.2010.519050 (cit. on pp. C97, C105).

[4] G. N. Mercer and R. O. Weber. "Combustion waves in two dimensions and their one-dimensional approximation". In: Combust. Theor. Model. 1 (1997), pp. 157-165. DOI: 10.1088/1364-7830/1/2/002 (cit. on pp. C97, C98).

[5] J. J. Sharples, H. S. Sidhu, A. C. McIntosh, J. Brindley, and V. V. Gubernov. "Analysis of combustion waves arising in the presence of a competitive endothermic reaction". In: IMA J. Appl. Math. 77 (2012), pp. 18-31. DOI: 10.1093/imamat/hxr072 (cit. on pp. C97, C100, C101, C105).

[6] S. D. Watt, R. O. Weber, H. S. Sidhu, and G. N. Mercer. "A weight-function approach for determining watershed initial conditions for combustion waves". In: IMA J. Appl. Math. 62 (1999), pp. 195-206. DOI: 10.1093/imamat/62.2.195 (cit. on pp. C96, C102).

\section{Author addresses}

1. S. D. Watt, School of Science, UNSW Canberra, Canberra 2600, Australia

2. Z. Huang, School of Science, UNSW Canberra, Canberra 2600, Australia

3. H. S. Sidhu, School of Science, UNSW Canberra, Canberra 2600, Australia 